\title{
Development of a Wall-Sticking Drone for Non-Destructive Ultrasonic and Corrosion Testing
}

\author{
Rami A. Mattar* (D) and Remy Kalai \\ Department of R\&D, Amerapex Corporation, Houston, TX 77092, USA; rkalai@amerapex.com \\ * Correspondence: rmattar@amerapex.com; Tel.: +1-713-263-0900
}

Received: 23 January 2018; Accepted: 21 February 2018; Published: 24 February 2018

\begin{abstract}
Refineries' structures require constant inspection, maintenance of their structural health condition, and safety of the users; however, accessing these structures is getting more and more difficult due to their enormous height and size. In order to deal with this problem, many researchers have developed several robots for wall crawling, yet there is no guaranteed solution. One of the critical reasons why existing wall-crawling robots have not been available in the field is the risk of accidental fall due to operational failure from the harsh environment, like strong wind and the surface's unpredictable condition. Therefore, we attempted to develop a wall-sticking aerial robot platform that can approach any place of the structure by flying and sticking to the target place. The robot is equipped with electro-magnetic hold mount elements to stick the sensor probe on the surface of the structure. This paper deals with installing the wall-sticking mechanism on the aerial robot.
\end{abstract}

Keywords: drone; nondestructive testing; NDT; wall-sticking; UAV; contact based inspection

\section{Introduction}

Drones have become increasingly autonomous with their services and usability. Drones started as a consumer and hobbyist phenomenon, but more recently they have grown into the field of remote visual inspection of industrial assets and sensing and other enterprise use cases. According to [1], last year alone, drone startups saw more than $\$ 450$ million of investments. It is a great addition to the inspection methods that are utilized at the moment in the inspection industry. Drone inspections are innovative inspection methods. The customer demand is growing rapidly and the possibilities are developing daily. Demand is significantly higher for military applications, although commercial applications are gradually catching up.

While non-contact-based drone inspection, such as visual, optical, IR, LIDAR, and gas detectors etc., is moving quickly to a commodity business, significant efforts are still to be made in aerial robotics and nondestructive testing (NDT) measurement technology to access conventional contact NDT, such as ultrasound testing (UT) and eddy-current testing (ECT).

There are only a few studies available where drones are utilized to monitor structures, which are only visual and image processing-based methods.

The objective of the project is to develop breakthrough industrial inspection solutions integrating the most recent robotics technologies. In the most challenging deliverables of the project, the consortium will deliver industrial aerial robots able to perform contact NDT, such as UT and ECT, leveraging the miniaturized, wireless inspection technologies of Amerapex NDT Inspection, Houston, TX, USA.

Aboveground storage tanks throughout their operating life are subjected to considerable operational and environmental forces, and subject to corrosion and cracks on the surface and subsurface level that travel parallel to the surface.

Usually cranes, scaffolds, rope-access, and people are used to inspect both the storage tanks and the overall structure. Maintenance and inspection are, thus, costly, time consuming, and risky for those who carry out the inspection. The solution to this can be to let autonomous drones do the work. 
Inspection robots play an important part in the oil and gas industry by taking the invaluable role of inspection, monitoring, and surveillance of complex structures in the industries, and, thereby, averting any disasters that may occur. The use of robots help in reducing human intervention, increase operational efficiency, reducing costs, and improving safety.

\section{Concept of a Wall-Sticking Drone}

Wall sticking of the aerial robot can be accomplished by the combination of the thrust force and wheel drive force with maximized friction between the drone wheel and the surface [2,3]. However the authors of this paper have demonstrated the wall sticking of an unmanned aerial vehicle by the combination of the thrust force and electromagnetic force to press the sensor probe on the metal surface. If the friction coefficient is higher than 1 , the robot can stick to the vertical surface with the thrust force toward the wall $[4,5]$. The authors have investigated this mechanism using a simple tri-copter and conducted outdoor experimental tests. Figure 1 describes the principle of the drone wall-sticking mechanism in the industrial structure space.

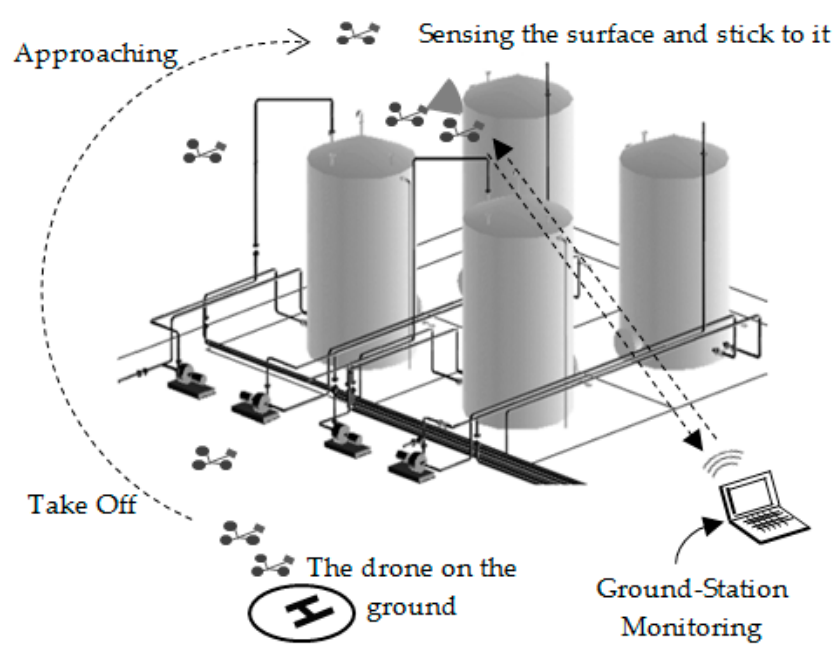

Figure 1. Diagram of an implementation of the system on a refinery tank farm.

\section{System Description}

The contact-based drone inspection was studied to evaluate the possibility of conducting ultrasonic thickness testing at random spots on structures that are not immune to corrosion and material degrading due to processes and other environmental impacts, such as storage tanks in refineries and petrochemical plants. It is meant for attaching the ultrasonic sensor probe to an open-source robotics vehicle platform (see Figure 1).

While designing the system, the primary design objectives kept in mind are: ease of use, modularized for fast deployment of sensors, real-time data display and, most importantly, meet the safety codes and regulations for hazardous environments.

The drone is a vertical take-off and landing, or VTOL, vehicle. It was selected from a set of criteria that met our needs:

1. Cost and availability.

2. Spare-parts and reparability.

3. Open-source hardware and software.

\subsection{Frame Design}

The UAV is a tri-copter and the goal is that the yaw functions differently. The rear motor pivots giving yaw more like a helicopter instead of like a quad-copter, which uses differential torque to 
achieve yaw. Differential torque is much weaker and slower. It works assuming that the system is basically in balance and only a slight change in torque will yield yaw. Pivoting the rear motor will yield more powerful yaw control (see Figure 2).

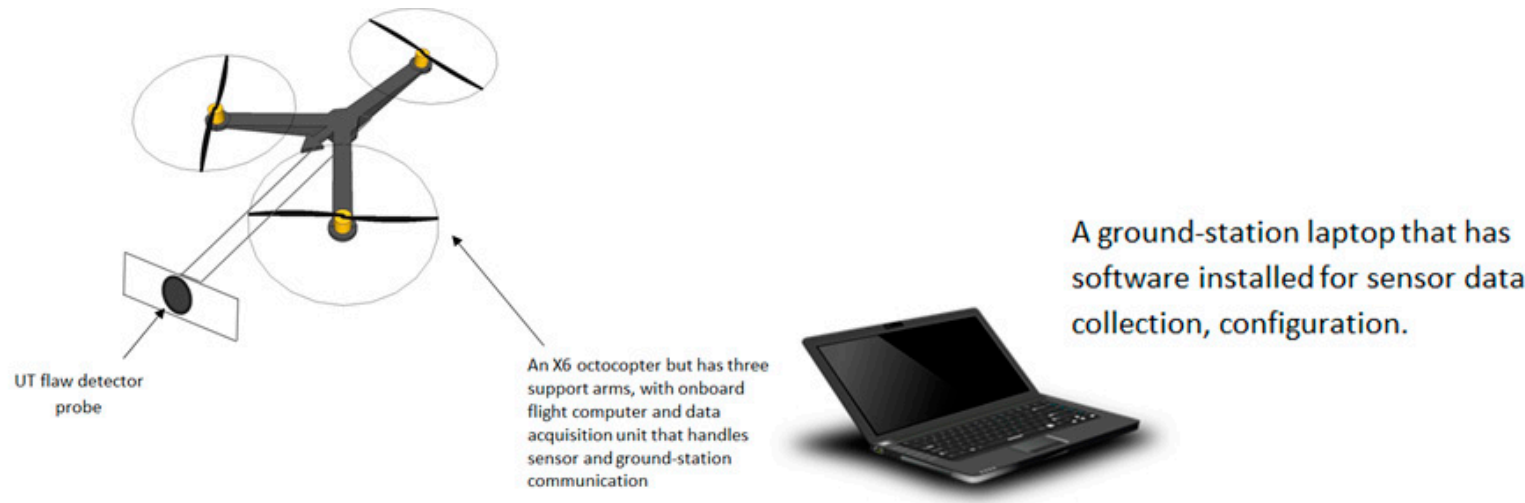

Figure 2. NDT ultrasonic testing UAV system overview.

The main materials of the UAV frame are carbon fiber plates. The selection of motors was based on the frame size, propeller size, battery capacity, and motor drive. Figure 3 shows the UAV general system block diagram.

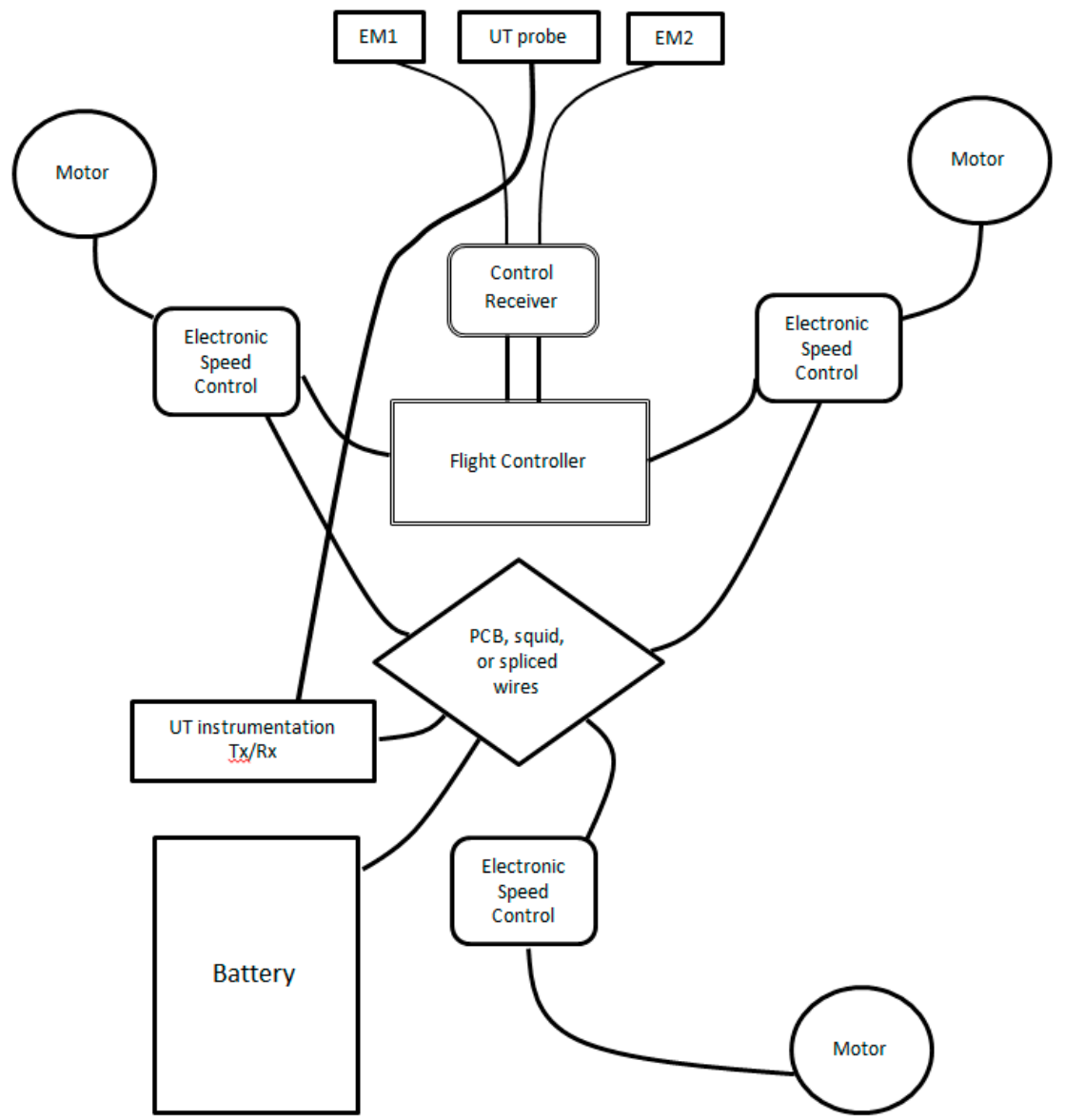

Figure 3. UAV general system block diagram. 
The UAV itself was designed around this application. The tri-copter has much more "yaw" control than a standard quad and it handles the wind conditions much better. Secondly, the forward motion is not significantly different; the yaw is the difference. Thirdly, the overall cost of the airframe is about the same as a quad-copter, because the extra servo and mechanism for yaw is about the same price as an extra motor setup. The rest of the frame is not that different from a quad-copter. One less leg does not appreciably change the material costs.

Interchangeable sensor modules were attached to the platform. Sensor data was relayed through the flight computer via a wireless telemetry link to the ground station app. The ground station application software displays the sensor data in real-time.

It consists of Y Copter access panel (S), tri copter battery tray, landing gear plates, Y Copter frame, Motor mount for pivoting motor, M3 and M4 lock nut retainer plates, servo mount, stationary motor mounts, servo mount carbon tab (ENV), GPS sensor, 16,000 mAH battery, receiver/transmitter for RC, ESC's for motors, and a Pixhawk control module.

The platform supported a simple and expandable interface for attaching custom sensors to the drone, overcoming the limitation of single-purpose platforms which are costly to convert for other tasks. Since the sensor system is modularized, sensors can be exchanged rapidly. In addition to the hardware platform, which easily integrates sensors, it is possible to use an established open-source infrastructure to collect sensor probe data from the drone in real-time. The drone need not be visible to the operator, but some line-of-sight is, however, required to ensure the signal is not dropped and real-time data lost.

The sensor gauge module with its transceiver unit is fitted inside the canopy and it can be fitted to the bracket in various ways. It will not interfere with the structural integrity of the drone as long as the module is kept within certain physical limits.

\subsection{Mechanism of Wall-Sticking}

The UAV has a capacity of hovering like most common drones, and is manually controllable by a remote operator by means of a remote control, or operate partially automated. It restrains a fixed extension arm that extends far from the neighboring blades with a mounting plate that contains compact and lightweight articulating flexible coils (see Figure 4).

During a data acquisition by the drone, it may, for example, based on the accuracy of the steering and hovering by the operator, and/or under the effect of a side wind or a stream of air, undergo rotational movements about its axis of pitch, such as vertical movement (up or down) and/or side movement (right or left).

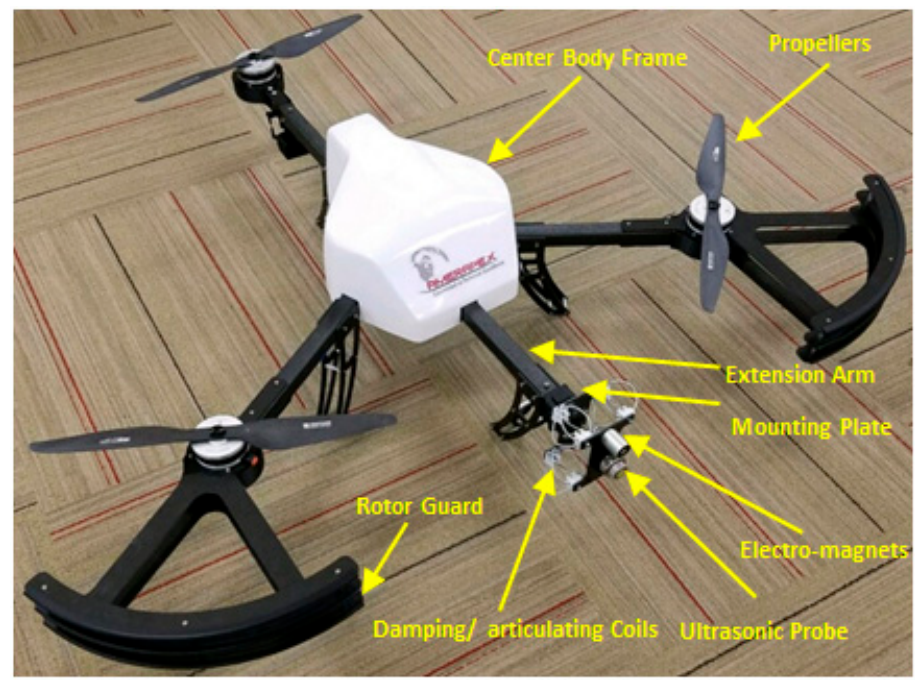

Figure 4. The drone and its components. 
Such events may cause the detachment or removal of the drone's sensor probe from the surface under testing and prevent it from taking the inspection readings. According to an optional feature of the invention, the articulating joint or (coil) may assist in absorbing the impact when the UAV flies directly to the surface of interest and lands on the probe side (front side of the UAV) to stick on it. By the combination of the thrust force generated by the UAV and the electromagnet units that surround the probe, the pressurization of the sensor probe against the surface will be achieved. The motors are AC brushless motors that offer high torque and efficiency.

Front rotor guards are designed to protect them from contact with the wall, which is also made from carbon fiber. The ultrasonic probe takes a measurement when applied against a surface. It is held stationary for a few seconds ( 1 to $2 \mathrm{~s}$ ) for a measurement to be taken. This allows measuring of the thickness of the metal surface under examination per the ASNT 'Ultrasonic Testing Standards and Practices'. The ultrasonic probe induces a normal beam ultrasonic signal that travels through the surface and subsurface, and the reflected signal from the back surface is detected by the probe and is converted to a digital reading of the thickness of the part underneath the probe.

The test was initially conducted with the sensor onboard without any kind of magnet. Then, to keep it from moving on the surface, we added a small permanent magnet. However, the basic concept of just pushing against the surface with the drone will work without the drone immediately hovering uncontrollably. The controls eventually had an issue because of the heading component in the control logic. This shows up in the rudder/yaw performing the wrong move a few times. The last time it was disconnected from the wall to prevent any further issues. To make this work long-term a decision has been made to modify the flight control code to put it in a "rate" gyro mode when pushing against the wall. This would prevent a buildup of control and allow the drone to steadily push against the wall without any significant issue. We tested with a light, flexible permanent magnet (shown in Figure 5), suggesting it might work. Its adhesive force is $32 \mathrm{lbs} .$, has a width of $1{ }^{\prime \prime}$, and it weighs $3.3 \mathrm{oz}$.

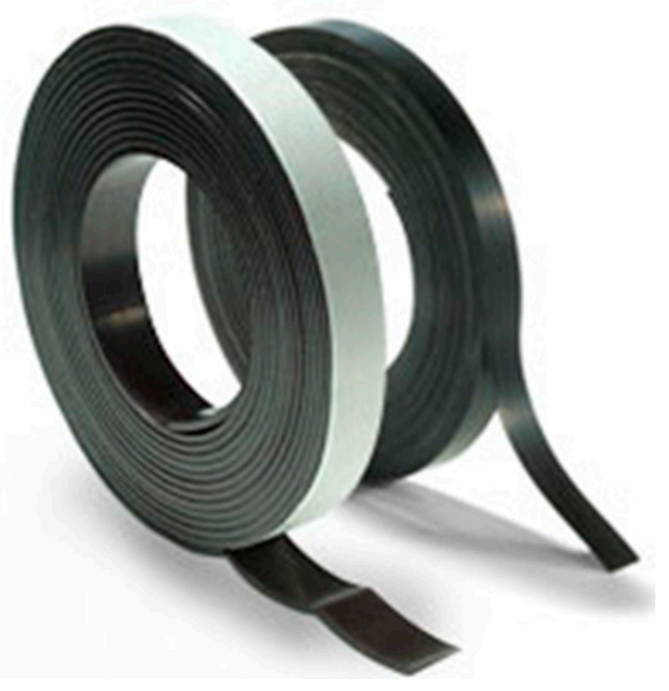

Figure 5. The flexible magnetic material.

The flexible magnetic material was wrapped around the mount frame of the probe, as shown in Figure 6. However, the test flight shows we can push the UAV against the wall and it stays relatively stable without the permanent magnet. The permanent magnet did not show any significant help to sticking the probe on the wall, as we determined that the magnet's surface contact area is not sufficient enough to generate the needed traction force. The effective force would be generated by a larger permanent magnet, but that will be bulky and heavy, hence, not feasible for this purpose. 


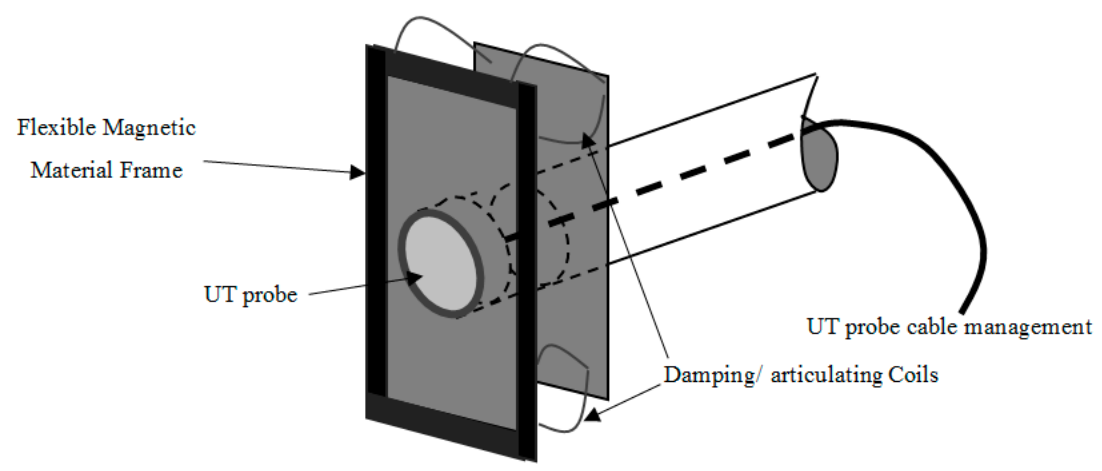

Figure 6. The flexible magnetic material wrapped around the probe mount.

To achieve the sticking force and stationary level needed to take a reliable reading, two electromagnets (EM) are suggested. EM combines the advantages of electro- and permanent magnets.

Each EM used is a round design that provides adhesive forces of up to $120 \mathrm{lbs}$. They are made of a zinc-plated case, $1.5^{\prime \prime}$ diameter $\times 1.5^{\prime \prime}$ long - weight $10 \mathrm{oz}$. The electrical specifications are: \#20 AWG lead wires $\times 24$ " long outside of the magnet, 12 VDC, 4.0 watts, and $100 \%$ duty cycle standards.

The device creates a very strong magnetic contact with a ferrous target, and it supports the UAV and $\mathrm{RC}$ remote control.

An ON command results in achieving full magnetization.

- An OFF command results in releasing or unsticking the unit from the surface. The ultrasonic probe and both EMs are housed in one aluminum plate, as shown in Figure 7.

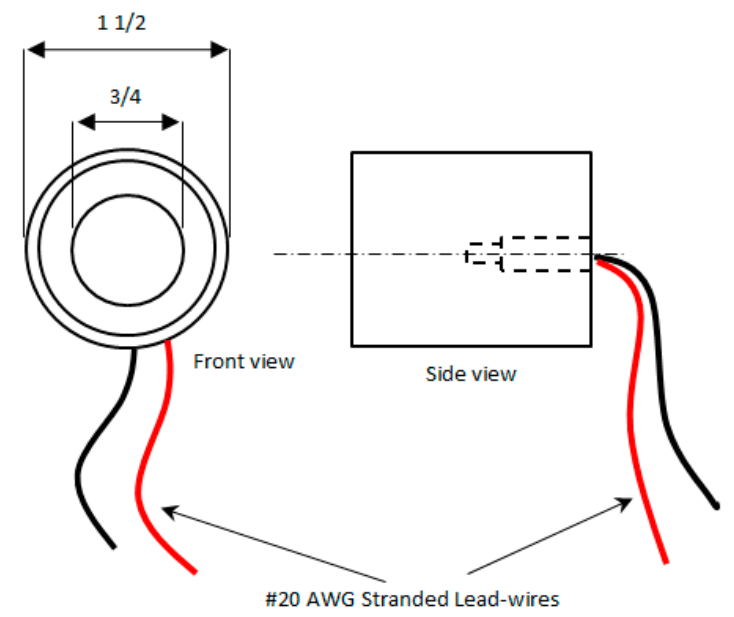

(a)

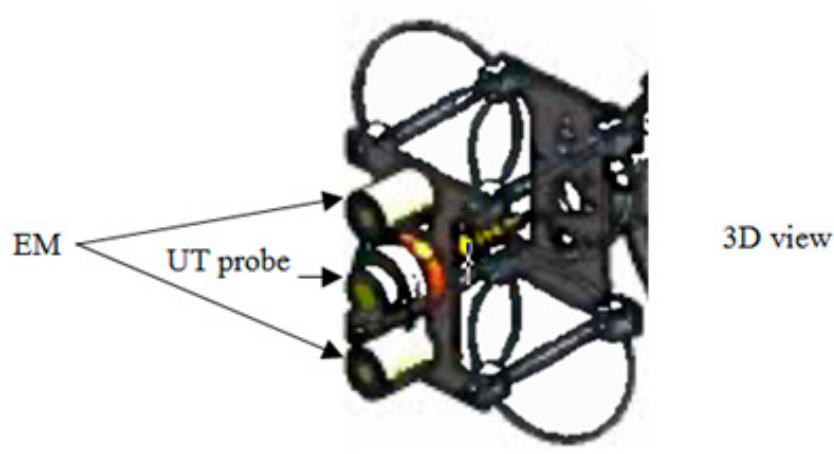

(b)

Figure 7. (a) EM schematic, and (b) the ultrasonic probe and EMs with the articulating joint design. 
The ultrasonic thickness gauge is a commercial off-the-shelf unit that is designed for common thickness gauging applications with the added benefit of being able to store measurements within the gauge. All ultrasonic thickness gauges should be calibrated to the velocity of sound of the material being measured. Coatings have a different velocities of sound than metal and it is important they are not included in the measurement. Multiple echo ensures all coatings, up to $6 \mathrm{~mm}$ thick, are completely eliminated from the measurement. The probe used is a $2.25 \mathrm{MHz}$ probe that works well on heavily-corroded metal. Its resolution and accuracy are $0.1 \mathrm{~mm}(0.005 \mathrm{inch})$ and $\pm 0.1 \mathrm{~mm}$ (0.005 inch), respectively. A transmitted ultrasound pulse travels though both the coating and the metal and reflects from the back wall. The returned echo then reverberates within the metal, with only a small portion of the echo travelling back through the coating each time. The timing between the small echoes gives the timing of the echoes within the metal, which relate to the metal thickness. The gauge will interpret the echoes automatically and calculate the thickness. The measuring range of a $2.25 \mathrm{MHz}$ probe goes down to $3 \mathrm{~mm}$, which is perfectly acceptable in most applications. This technique is referred to as the automatic measurement verification system (AMVS) (see Figure 8).

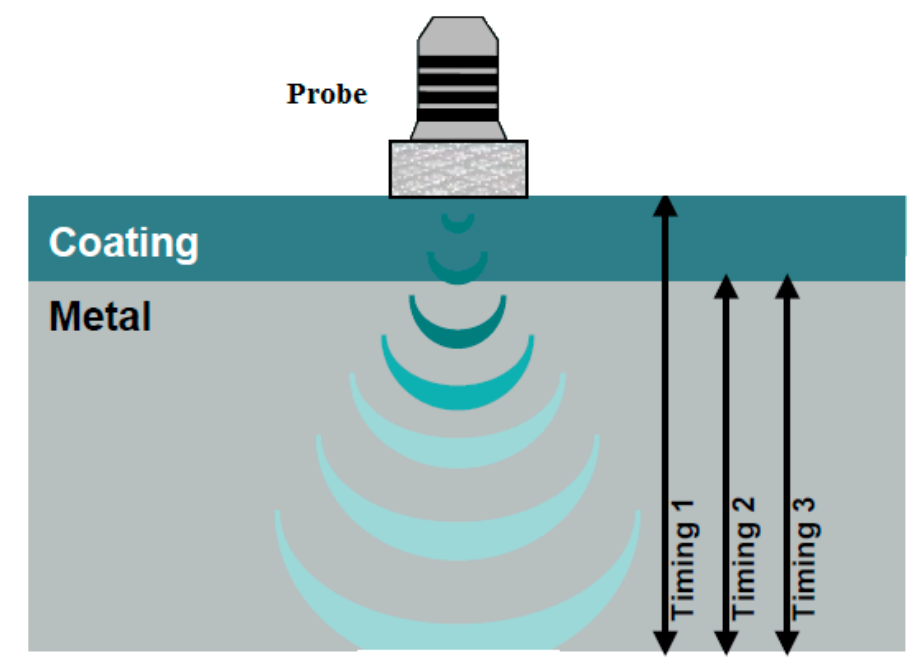

Figure 8. The deployed ultrasonic probe and testing method.

Lithium-ion batteries (16,000 MAH) used to power the system are lightweight, and have a large capacity, high discharge rate, and good energy storage to weight ratio. The readings picked up by the sensor module are then relayed to a ground station using the telemetry modules; on the drone, the radio link module is connected directly to the flight computer.

The author has attached a dry couplant to the probe for contact inspection. The dry couplant (elastomer) is designed specifically for ultrasonic inspection applications. Unlike dry couplants normally used as an integral part of ultrasonic probes, this elastomer can be applied independently of the probe. Acoustic impedance of the material is nearly the same as water and its attenuation coefficient is lower than all other documented elastomers and many plastics.

\section{Discussion of Related Work}

As mentioned in the introduction, there were only a few studies available where drones are utilized in the inspection of structures, which are focused on visual inspections only. Many companies are able to achieve NDT visual inspections using drones. Companies, such as Industrial Works, are currently able to identify issues, like water accumulation, solar loading, and areas susceptible to rust and corrosion, which cannot be seen by the human eye. [6] These drones are able to achieve the same results as the drone proposed by the authors when performing visual inspections, yet they are unable to make contact inspections. However, one company has been able to achieve similar 
results as the drone manufactured by the authors. The Center for Advanced Aerospace Technologies (CATEC), a technology company in Spain, has created a drone capable of making contact with surfaces at altitude and using a probe to take measurements. The drone has similar features to hover at altitude. The main difference is the mechanism to make contact with a surface. Where the drone created by the authors uses electromagnets to make a strong connection with the surface, CATEC uses three prongs to push into the surface and make contact. Comparing their drone with the proposed concept will not make as strong a connection with the surface because of the proposed concept of utilizing the power of the electromagnets rather than just pressure created by the drone. $[7,8]$. Ellenberg et al. performed an investigation on remote sensing capabilities of a commercialized drone (Parrot AR 2.0) for crack detection from various distances [9]. An algorithm was developed for post-image processing where a field test was conducted on a bridge in order to evaluate the performance of the drone. Sankarasrinivasan et al. introduced an approach involving a combination of the Top-hat transform and HSV (hue, saturation, and value) thresholding technique which is a tool for solving clustering problems in image processing for detecting cracks, using a drone for a real-time SHM [10]. Another related work was executed by Vel Tech University in India. It was a field testing that was conducted in an outdoor environment to evaluate the performance for the proposed study, during existing factors, such as wind, and random image noises that resulted in a few inaccurate results. Eschmann et al. used an octocopter for a building inspection where photos were taken at high speed and frequency [11]. According to their study, more than 12,000 photos were taken over a four-day period for an inspection of cracks on the target structure. Markus Eich et al. from the Robotics Innovation Center at Bremen, Germany, have created a magnetic wall climbing robot that is capable of attaching to ships in order to perform visual inspections. Their robot is able to reach heights exceeding $10 \mathrm{~m}$ [12]. Amit Shukla et al. also implemented the use of UAVs in detecting corrosion in oil and gas pipelines [13]. This UAV is used in visual inspections of pipelines from low altitudes with the intention of reducing the risk of having humans do these inspections. Lee et al. have developed a crawling magnetic robot that can navigate in a tubular environment, and a magnetic pulley module is utilized to generate a drilling motion to unclog blocked regions and uncover motions of a stent cover for a self-expandable stent deployment [14]. Na et al. proposed and performed a concept of converging a drone with a vibration-based non-destructive evaluation method [15]. Their technique requires one to permanently attach a PZT transducer onto the surface of the target structure, usually in $10 \mathrm{~mm}$ square sizes. They used a PZT with a frequency range between 20 and $400 \mathrm{kHz}$, to examine the mechanical impedance of the host structure. However, according to their findings, if the host structure is non-metallic (e.g., concrete, composite, wood, etc.), a support permanent magnet must be attached to the host structure permanently.

\section{Test and Result}

The author initially used a simpler and smaller version of the tricopter for testing while fine-tuning the flight control codes in order to minimize damage costs to the larger unit that is equipped with the sensing device. The goal is to have the control logic used during normal flight to be the same as the control logic that is used when the drone is against the wall.

The author has initially conducted manual tests around the structure to collect data as a reference.

The drone tests have shown that the drone takes off from the ground and hovers near the target area with high stability, and sticks to it using the EMs and back pressure from the drone itself.

As seen in the hovering scenarios in Figure 9, the different scenarios show how the drone would maintain contact even with the existence of movement or instability due to high wind speed or other mechanical instability. The EM is powerful enough to hold the drone, and it has flexible ring-like coils at the corners of the mounting plate that enable it to pivot left, right, up, and down, while the sensor is continually making contact and not moving. 

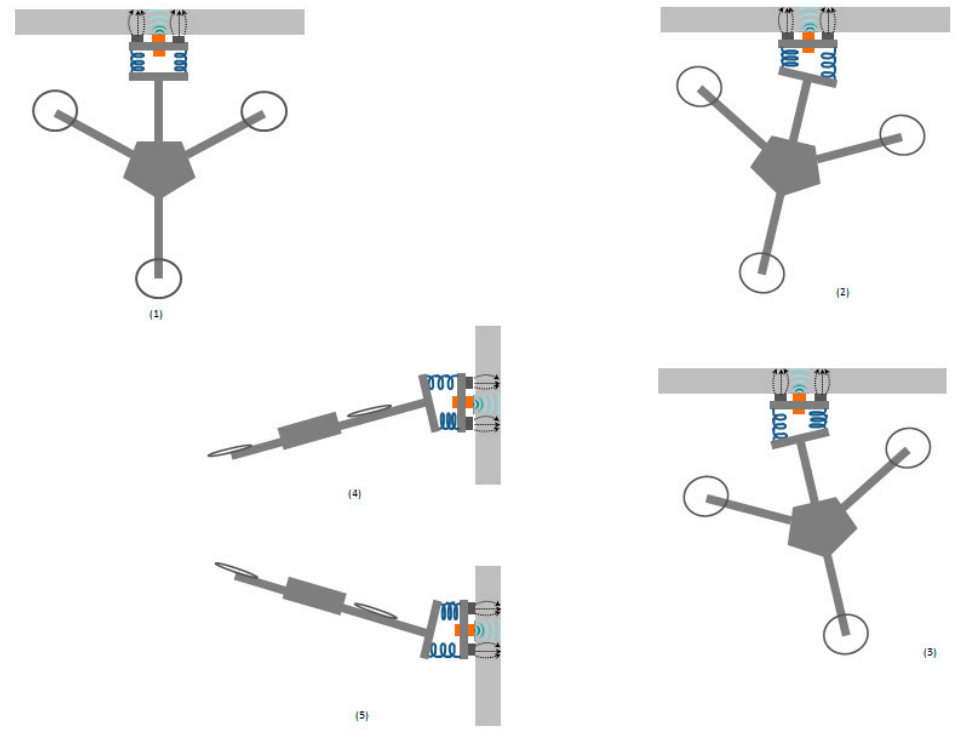

Figure 9. Different scenarios the drone experiences during wall-sticking.

In a real-world inspection demonstration on a local crude oil storage tank (nominal thickness $=0.2$ inches) at an oil well site, as shown in Figure 10a, the drone takes off and hovers to reach the target spot on the storage tank wall. When it is close to the target, the operator sends the TURN ON command to the EMs through the remote control to turn them on. The drone sticks to the wall, and the probe will induce the ultrasound as soon as it contacts the wall. It takes the measurement data, and displays it in real-time at the ground-station laptop (see Figure 10b). It can measure the thickness of the storage tank wall with up to \pm 0.005 inch accuracy. After receiving the data, the operator will send the TURN OFF command to the EMs to turn them OFF. The drone will then be free to fly to the next desired destination. The author attempted 10 flight tests per battery. Each flight took no more than $20 \mathrm{~s}$ (including take off, hover to the inspection spot, stick to the surface for inspection, and send the data wirelessly to the ground). The experimental test showed a success rate of wall-sticking without the EMs higher than $90 \%$, while with the Ems it was 100\%. Drone flight time reached up to 15-20 min per battery pack.

The wind speed was $22 \mathrm{mph}$, and the number of conducted tests attempts was 10 . The process is shown in Figure 10c where the thicknesses were measured 10 times manually versus using the drone.

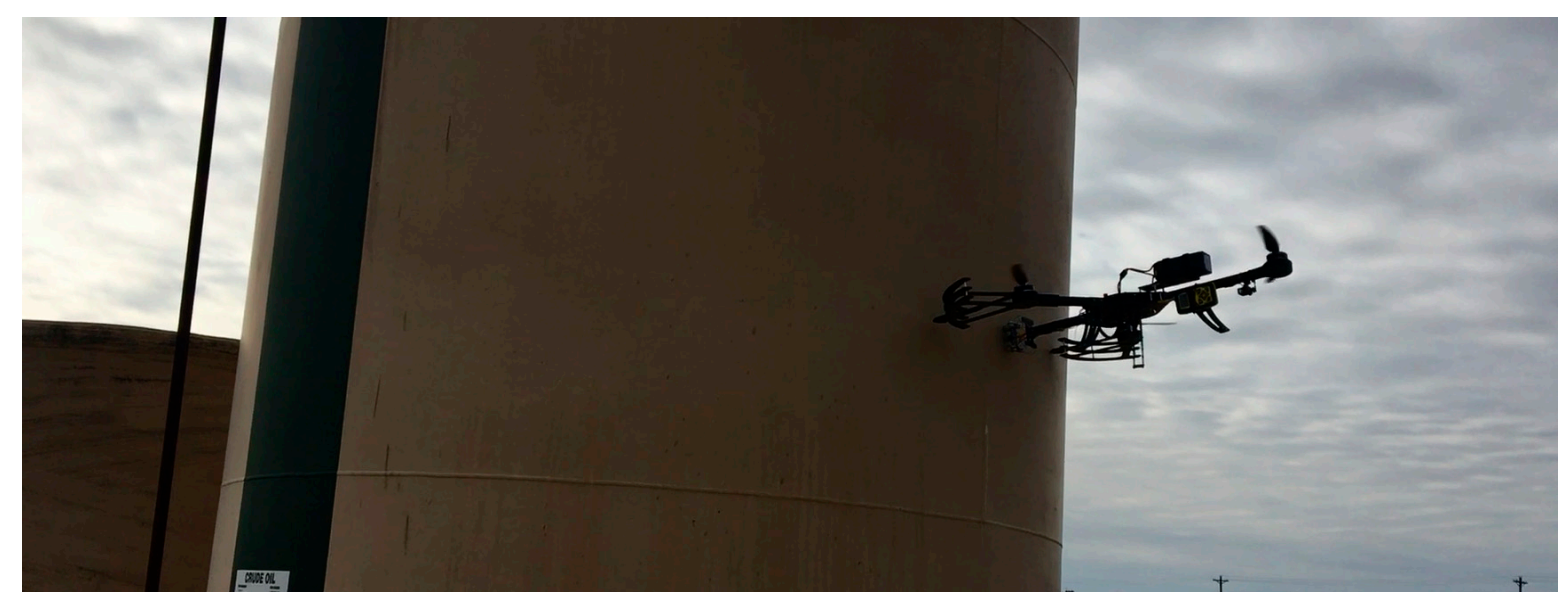

(a)

Figure 10. Cont. 


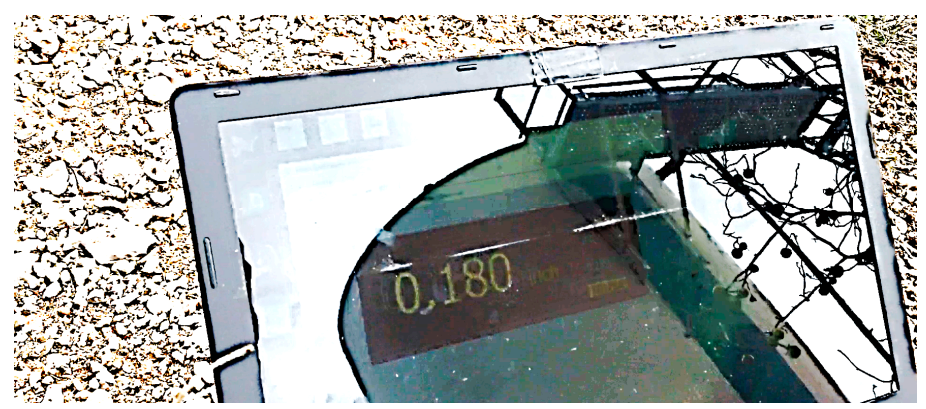

(b)

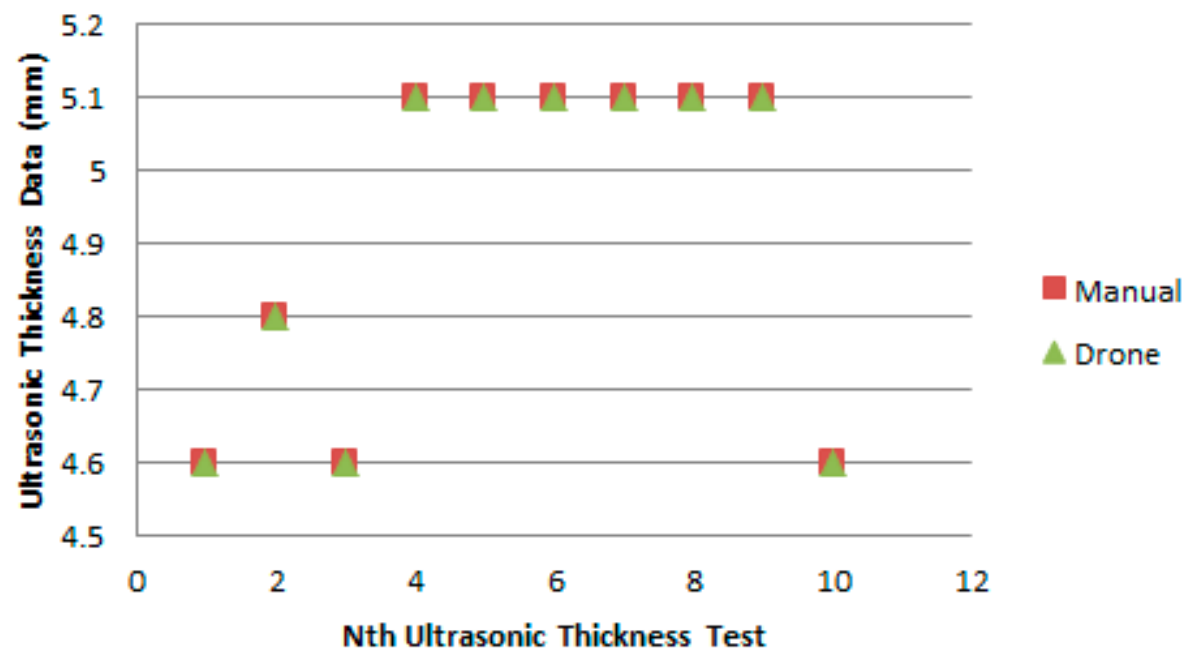

(c)

Figure 10. (a) A real-world inspection test demonstrates wall-sticking drone on a crude oil storage tank for random spots checks. (b) Thickness Measurement data displayed in real-time at the ground-station laptop. (c) Drone ultrasonic thickness data vs. manual data.

The result also showed that the collected measurement data ranged from 0.18 to 0.2 inches. Data from the drone matched the manually-taken data $100 \%$. Based on the author's findings, in cases where the target structure is coated with an anti-corrosion paint layer or any sort of coating layer that prohibits the EMs from being useful, there was still an ability to conduct the test successfully through the back pressure that is produced by the drone that holds the probe in place with good stability. This also applies to non-metallic surfaces, such as concrete, composite, and wooden structures, etc.

\section{Conclusions}

The authors have demonstrated the operability of automating relatively "low-skilled" manual labor that usually requires building scaffolds and/or rope access only to reach higher altitudes and hard-to-reach areas. The feasibility of the wall-sticking robot platform has been verified with a high success rate. The concept excels in the face of the current limitations of using a UAV in combination with a visual inspection method, and any contact-based method. From the test that was conducted in the laboratory and in the field, it was proved that the NDT ultrasonic testing method, and a number of other NDT methods, can be achieved utilizing a UAV.

Acknowledgments: This work was financially supported by Amerapex Corporation, an oil and gas and NDT engineering company located in Houston, Texas. 
Author Contributions: Rami Mattar conceived, designed, and performed the experiments; Rami Mattar with the assistance of Remy Kalai researched the suitable hardware components for the designed system and analyzed the data; and Rami Mattar with the assistance of Remy Kalai wrote and edited the paper.

Conflicts of Interest: The author discloses that the intellectual property described in the abstract is owned by Amerapex Corporation. The study was funded by Amerapex Corporation, the employer of author Rami Mattar. Author Remy Kalai is a contractor for Amerapex Corporation.

\section{References}

1. The Inspectioneering Journal. Available online: https://inspectioneering.com/news/2016-04-18/5276/ american-petroleum-institute-supports-new-drone-technology (accessed on 10 October 2017).

2. Glez-de-Rivera, G.; Garrido, J.; Ponticelli, R. Design considerations of a small UAV platform carrying medium payloads. In Proceedings of the 2014 Conference on Design of Circuits and Integrated Circuits (DCIS), Madrid, Spain, 26-28 November 2014.

3. Shin, J.U.; Kim, D.; Kim, J.H.; Myung, H. Micro-aerial vehicle type wall climbing robot mechanism for structural health monitoring. In Proceedings of the IEEE SPIE 2013 (Smart Structures and Materials + Nondestructive Evaluation and health Monitoring), San Diego, CA, USA, 10 March 2013.

4. Xiao, J.; Wang, H.J. Contemporary Issues in Systems Science and Engineering; CHAPTER22: Advances in Climbing Robots; Wiley, IEEE Press: Austin, TX, USA, 2015.

5. Myeong, W.C.; Jung, K.Y.; Jung, S.W.; Jung, Y.H.; Myung, H. Development of a drone-type wall-sticking and climbing robot. In Proceedings of the 12th International Conference on Ubiquitous Robots and Ambient Intelligence (URAI 2015), Goyang, Korea, 28-30 October 2015.

6. Industrial SkyWorks. Available online: http://industrialskyworks.com/oil-and-gas-drone-inspections / (accessed on 10 December 2017).

7. sUAS News, the Business of Drones. Available online: https://www.suasnews.com/2017/10/catecdevelops-aerial-contact-technology-inspection-drones / (accessed on 12 December 2017).

8. Center for Advanced Aerospace Technologies (CATEC). Available online: http:/ / www.catec.aero (accessed on 12 December 2017).

9. Ellenberg, A.; Branco, L.; Krick, A.; Bartoli, I.; Kontsos, A. Use of unmanned aerial vehicle for quantitative infrastructure evaluation. J. Infrastruct. Syst. 2014, 21, 04014054. [CrossRef]

10. Sankarasrinivasan, S.; Balasubramanian, E.; Karthik, K.; Chandrasekar, U.; Gupta, R. Health monitoring of civil structures with integrated UAV and image processing system. Procedia Comput. Sci. 2015, 54, 508-515. [CrossRef]

11. Eschmann, C.; Kuo, C.M.; Kuo, C.H.; Boller, C. Unmanned aircraft systems for remote building inspection and monitoring. In Proceedings of the 6th European Workshop on Structural Health Monitoring, Dresden, Germany, 3-6 July 2012.

12. Eich, M.; Vögele, T. Design and control of a lightweight magnetic climbing robot for vessel inspection. In Proceedings of the 2011 19th Mediterranean Conference on Control \& Automation (MED), Corfu, Greece, 20-23 June 2011.

13. Shukla, A.; Xiaoqian, H.; Karki, H. Autonomous tracking and navigation controller for an unmanned aerial vehicle based on visual data for inspection of oil and gas pipelines. In Proceedings of the 2016 16th International Conference on Control, Automation and Systems (ICCAS), Gyeongju, Korea, 16-19 October 2016.

14. Lee, W.; Nam, J.; Jang, B.; Jang, G. Selective motion control of a crawling magnetic robot system for wireless self-expandable stent delivery in narrowed tubular environments. IEEE Trans. Ind. Electron. 2017, 64, 1636-1644. [CrossRef]

15. Na, W.S.; Baek, J. Impedance-based non-destructive testing method combined with unmanned aerial vehicle for structural health monitoring of civil infrastructures. Appl. Sci. 2016, 7, 15. [CrossRef]

(C) 2018 by the authors. Licensee MDPI, Basel, Switzerland. This article is an open access article distributed under the terms and conditions of the Creative Commons Attribution (CC BY) license (http://creativecommons.org/licenses/by/4.0/). 